\title{
Experience of Joy and Sadness in Alexithymic Emotional Discourse
}

\author{
Marie-Mathilde Dupont-Leclerc ${ }^{1}$ \\ Serge Lecours \\ Université de Montréal
}

\begin{abstract}
Alexithymia is a personality trait characterized by difficulties in identifying and describing emotional feelings. Impairment in the cognitive processing of emotions experienced by individuals with alexithymia translates into a limited capacity to symbolize their emotions. However, despite being one of the core aspects of the construct of alexithymia, emotional elaboration has not been thoroughly investigated. While a few studies have reported quantitative features of alexithymic individuals' discourse, the qualitative properties of alexithymics' emotional discourse and the difference in symbolization between positive and negative emotions remain to be examined. This study aims to explore how individuals with high levels of alexithymia symbolize their subjective emotional experiences, through an investigation of specific features in their discourse pertaining to positive and negative emotions. The sample consisted of 9 individuals with high levels of alexithymia, screened with the TAS-20. The participants were interviewed about a typical experience of joy and sadness. Data were analyzed using an interpretative phenomenological analysis approach. Themes associated with sadness revealed that high-alexithymia individuals tend to avoid contact with sadness and to perceive sadness as a state imposed by external events. Themes associated with joy revealed that this emotion seems easier to share with peers. Moreover, joy appeared to be easier to express and symbolize for highalexithymia individuals than sadness. This comprehensive description of alexithymic individuals' emotional discourse provides us with a better understanding of the symbolization of emotions according to their valence and allows us to better recognize the ways in which individuals with high levels of alexithymia express their emotions.
\end{abstract}

\section{Introduction}

The term alexithymia was introduced by Sifneos in 1972 to frame clinical cases of psychosomatic patients who experienced significant difficulty in using language to express emotions, a dearth of fantasy life, and a lack of symbolic dreaming (Sifneos, 1994). Today, alexithymia is measured by the Toronto Alexithymia Scale (TAS-20) as a personality trait composed of three main characteristics: (i) a difficulty identifying and distinguishing between feelings and bodily sensations of emotional arousal, (ii) a difficulty describing one's feelings to others and (iii) an externallyoriented style of thinking with pragmatic contents (Bagby, Parker \& Taylor, 1994).

${ }^{1}$ Correspondence concerning this article should be addressed to Marie-Mathilde Dupont-Leclerc, Department of Psychology, Université de Montréal, C.P. 6128, succursale Centre-ville, Montréal, QC, Canada, H3C 3J7. Email: mariemathilde.dupont-leclerc@umontreal.ca

Language and Psychoanalysis, 2018, 7 (1), 62-83.

http://dx.doi.org/10.7565/landp.v7i1.1583 
These characteristics represent the manifestations of two main deficits. The first is an impairment in emotional awareness, which causes the emergence of the first two characteristics. The second deficit, termed "operative thinking" (pensée opératoire), is defined by a lack of fantasy associated to a concrete cognitive style, which leads to the manifestations of the third characteristic (Bagby, Taylor, Parker \& Dickens, 2005). Generally speaking, the characteristics comprised by the construct of alexithymia are considered to reflect a deficit in the cognitive processing of emotions (Sifneos, 1994) and therefore, in the symbolic representation of emotions (Lane et al., 1996).

Symbolization is the capacity to link emotions to various types of cognitive representations, to create links between these representations in order to form a complex schema, and to verbalize these links and the system they create. Thus, the construct of alexithymia is not characterized by an absence of somatic manifestations of emotions; rather, emotions are felt but are seldom verbalized (Zimmermann, Salamin \& Reicherts, 2008). Depending on the degree of alexithymia, this type of psychosomatic disconnect can be compared to emotional agnosia (not knowing or not recognizing emotions) or anomia (lack of words for feelings) (Taylor, Bagby \& Parker, 2016), two conditions in which the ability to adequately link non-verbal emotional symbols (e.g., emotions) with verbal organization (e.g., representations or associations of representations) is impaired (Sifneos, 1994). Nevertheless, emotional experiences must be symbolized in order to be integrated into psychic life (Lecours, Bouchard, St-Amand \& Perry, 2000) and to foster an effective regulation of emotions.

Since alexithymia is conceptualized as a deficit in the symbolization of emotion coterminous with felt emotions, it seems essential to examine the manner in which high-alexithymia individuals integrate experiences of emotions into their psychic lives. Discourse analysis is a powerful tool to evaluate three abilities related to the integration of emotions. Firstly, the approach allows us to analyze, through individuals' discourse, available symbolization resources used to articulate verbally affective experiences (Lecours \& Bouchard, 2011). Secondly, emotional discourse can be in and of itself an emotion regulation strategy; for example, by sharing a difficult emotional experience in order to elicit social support (Bagby \& Taylor, 1999a). Accordingly, this also allows us to analyze a variety of emotion regulation strategies. Lastly, discourse analysis allows us to examine the cognitive treatment of emotions (Luminet, Rimé, Bagby \& Taylor, 2004) and subsequently, representational deficits, which compose one of the core features of alexithymia. Despite being a central characteristic of the alexithymic experience, the quality of emotional discourse in alexithymic individuals has not been thoroughly empirically evaluated (Lecours, Robert \& Desruisseaux, 2009). Consequently, analyzing the emotional discourse of high alexithymia individuals appears to be a crucial step in order to better understand cognitive symbolization and emotion regulation deficits experienced by individuals with alexithymia.

\section{Emotional Discourse in Alexithymic Individuals}

The earliest descriptions of alexithymic discourse come from incomplete clinical observations. For example, Bagby and Taylor (1999a) described some patients capable of labelling their emotions, although they were incapable of either elaborating

Language and Psychoanalysis, 2018, 7 (1), 62-83. 
on them or linking them to memories or fantasies. McDougall (1978) emphasized that alexithymic individuals tend to use language as an act as opposed to a symbolic means of communicating feelings. For instance, language would be used to elicit a reaction in others instead of expressing and sharing an emotional experience.

From a more empirical point of view, the beginnings of alexithymic discourse analysis were mainly based on quantitative elements of verbal content. In written essays, Páez, Velasco, and González (1999) found negative correlations between high alexithymic scores and levels of introspection, the frequency of self-references, and the ratio of positive words used in describing positive emotions. Roedema and Simons (1999) also found that high alexithymic subjects used less affective words to describe their state than their control counterparts. Also, a study by Luminet et al. (2004) measuring the proportion of emotional words used by alexithymic individuals, suggested that the deficit in the use of emotional words would be present only in emotionally charged contexts. Thus, it would not be considered a deficit in accessing emotional vocabulary. Although these studies generally demonstrated a negative relationship between the frequency of emotional references and the degree of alexithymia, their relevance is only partial as they fail to address the level of cognitive organization of affects associated with the personality construct (Lecours et al., 2009). However, a more recent study attempted to address the level of cognitive organization of affect by analyzing the semantic conceptual level of emotion representation, through the number of recurrence of certain language patterns and knowledge related to emotion in individuals with high levels of alexithymia (Wotschack \& KlannDelius, 2013). The study's findings revealed the presence of a reduced differentiation and elaboration of emotion schemata in high-alexithymia individuals compared to low-alexithymia individuals, including a significantly lower use of words to conceptualize emotions (emotion words), fewer physiological-expressive terms, fewer synonyms, and a heightened level of hesitation and of null responses. The study concluded that alexithymia moderates language use by demonstrating that semantic differentiation of emotion is reduced in individuals with high levels of alexithymia. While the study by Wotschack and Klann-Delius (2013) addressed the conceptual level of emotional representation in high-alexithymic individuals, it has not examined their ability to put emotions into words, and the subjective aspect of the speech of high-alexithymia individuals.

More recently, some studies have taken an interest in the formal aspects of the discourse of alexithymic individuals in order to better understand notions related to the mentalization of affect (Lecours et al., 2009).

A first instrument, the Verbal Elaboration of Affect Scale (GÉVA) (Lecours et al., 2009), measures the mentalization of affect by evaluating the levels of tolerance and abstraction of verbalized affects. The use of the measure with individuals with high levels alexithymia also revealed that high-alexithymia discourse, when compared to low-alexithymia participants' discourse, is characterized by a diminished level of verbal elaboration of affects (Lecours et al., 2009). Furthermore, the spontaneous verbalization of affect and the use of references to emotions have also been demonstrated to be reduced within this population (Lecours et al., 2009).

A second instrument measuring formal aspects of discourse is the Level of Emotional Awareness Scale (LEAS) (Lane, Quinlan, Schwartz, Walker \& Zeitlin, 1990). Based

Language and Psychoanalysis, 2018, 7 (1), 62-83.

http://dx.doi.org/10.7565/landp.v7i1.1583 
on a piagetian framework, it assesses four hierarchical levels of emotional representation, and distinguishes between the two lower levels termed implicit and the two higher levels termed explicit. The higher the level achieved, the more elaborate the emotional awareness of the individual tested. Based directly on verbal expression, the lowest score is attributed to individuals who describe thoughts instead of emotions. The implicit levels are characterized by the descriptions of physical sensations, the use of general and undifferentiated emotional words (e.g., I would feel bad) or the descriptions of actions. The first explicit level includes individuals capable of differentiating typical emotions, and the second explicit level represents individuals capable of integrating various emotions to describe a nuanced psychological state. Hence, the discourse of individuals with low emotional awareness would be characterized by the use of vague emotional words, and descriptions of concrete actions and bodily sensations. A study found that the TAS-20 subscale "externallyoriented thinking style" is significantly, negatively correlated to the LEAS (Waller \& Scheidt, 2004), suggesting that only the cognitive component of alexithymia is related to lower levels of emotional awareness. Important conceptual and psychometric differences appear to exist between the concepts of alexithymia and emotional awareness, as shown by the overall lack of correlation between the total score of the TAS-20 and the LEAS (Subic-Wrana, Bruder, Thomas, Lane \& Köhle, 2005; Waller $\&$ Scheidt, 2004). It thus seems that the two concepts are partially overlapping, but measuring different aspects of emotional functioning (Taylor et al., 2016).

Finally, the Affect Consciousness Instrument (ACI) (Monsen, Eilertsen, Melgård \& Ødegård, 1996) measures the degree of consciousness and tolerance of affect, as well as the conceptual and non-verbal expression of emotions (Monsen et al., 1996). The dimension of conceptual expression of emotions evaluates the capacity to use clear, differentiated and nuanced language to describe a specific emotional experience. Hence, a low score of emotional consciousness refers to an individual who demonstrates difficulty in verbally describing and distinguishing emotions. This instrument's total score has been found to be significantly, negatively correlated with the "externally-oriented thinking style" and the total score of the TAS-20, but not significantly correlated with the LEAS (Waller \& Scheidt, 2004). This suggests, once again, a partial overlap with the alexithymia concept (Lecours et al., 2009) and the measurement of a different aspect of emotional functioning (Waller \& Scheidt, 2004).

While these findings provide a better understanding of the emotional organization underlying alexithymia, the instruments discussed above are only obliquely related to alexithymia. Although aspects of mentalization, emotional awareness and affect consciousness characterize a portion of the verbal behaviour associated with alexithymia, a large part of the alexithymic discourse remains unexamined.

\section{Symbolization of Positive and Negative Emotions}

Studies that have assessed alexithymic discourse quantitatively or formally have typically done so without differentiating between types of emotions, despite reports by clinicians mentioning a possible distinction in emotional symbolization based on specific emotions (Lecours, 2007). Indeed, some studies have reported that positive affects seem easier to mentalize than negative ones (Lecours \& Bouchard, 2011; Lecours et al., 2000). Differences appear to exist even within the class of negative

Language and Psychoanalysis, 2018, 7 (1), 62-83. 
emotions. For instance, deficits in symbolization of sadness have been considered more problematic as they are associated with more clinical symptoms and with borderline and narcissistic personality disorder traits (Bouizegarene \& Lecours, 2017; Lecours \& Bouchard, 2011).

With regards to alexithymia, the TAS-20 score has been found to be negatively correlated with positive emotions and positively correlated with negative affects in a number of studies with different samples (Lundh, Johnsson, Sundqvist \& Olsson, 2002; Taylor \& Bagby, 2013; Taylor, Bagby, Kushner, Benoit \& Atkinson, 2014). These findings are not surprising considering the strong association between alexithymia and anhedonia, as described by Krystal (1981). They suggest that alexithymia is not characterized by a homogeneous deficit, affecting all types of emotions in the same manner.

Despite these results, only a few studies (Luminet et al., 2004; Páez et al., 1999; Wagner \& Lee, 2008; Wotschack \& Klann-Delius, 2013) have measured positive and negative emotions separately within the discourse of individuals with high levels of alexithymia. One of these studies (Páez et al., 1999), found a significant negative association between the number of words used for positive emotions and the TAS-20 difficulty describing emotion subscale, but no significant association was found between the subscale and the proportion of negative emotion words. Another study, by Wagner and Lee (2008), which measured the verbal expression of positive and negative emotions separately for a positive and a negative event, found a significant negative correlation between the verbal expression of emotion and the TAS-20 total score, only if the emotion's valence was congruent with the event's emotion valence. These findings highlight the need to further investigate the differences in the symbolization of emotions based on the valence (positive or negative) of experienced emotions.

\section{Objectives and Research Questions}

Taking into account the aforementioned theoretical and empirical contributions, the present study's objective is to provide a more elaborate portrait of the qualitative aspects of the discourse of high-alexithymia individuals, in an effort to gain further insight into these individuals' subjective experiences of feelings. To meet this goal, an interpretative phenomenological analysis (IPA), grounded in a constructivist paradigm, will be conducted. The exploratory nature of the research requires an inductive approach specific to qualitative methods. Furthermore, the principle of coconstruction, underlying the constructivist paradigm, is an essential contribution to research on subjective experiences. Since high-alexithymia individuals tend to have a marked difficulty expressing their feelings, the dynamic between the researcher and the participant is fundamental because it can help the participant stay focused on, and verbalize his or her introspective experience of emotion. The researcher's participation in the construction of the participant's experience is also critical in facilitating inferences for understanding the symbolization process, which the participant may not be able to express alone.

The analysis of discourse will rely on experiences of joy and of sadness considering that the symbolization processes might differ with regards to positive or negative

Language and Psychoanalysis, 2018, 7 (1), 62-83.

http://dx.doi.org/10.7565/landp.v7i1.1583 
emotions. The comparison of positive and negative affect verbalizations will allow a better understanding of how deficits in the symbolization of emotion vary in relation to positive and negative emotions. Since symbolization deficits appear to be more strongly associated with negative emotions, an analysis of discourse on sadness will allow us to better recognize the deficits in the structure of emotional elaboration. Hence, the present study seeks to address the following research questions:

- $\quad$ How do high-alexithymia individuals describe emotional experiences of joy and sadness?

- What are the themes and formal characteristics of a typical emotional discourse in high-alexithymia individuals?

The second question has important clinical implications since the identification of typical characteristics the verbal productions of high-alexithymia individuals will facilitate the identification of these patients within clinical settings, and subsequently, will allow clinicians to tailor psychosocial interventions according to their specific needs.

\section{Method}

\section{Participants}

The study was based on interviews conducted with 9 participants who each provided narratives on sad and joyful experiences. Data saturation for the present IPA analysis has been reached using this sample. According to Smith, Flowers and Larkin (2009), the discourse analysis of 4 to 10 participants is most appropriate since it prioritizes the qualitative aspect of each individual experience. Therefore, this recommendation was followed. The participants were undergraduate psychology students, recruited from Université de Montréal. The sample was comprised of 6 women and 3 men, aged between 19 and 27 (mean age $=23$ years).

Alexithymia was measured with the TAS-20 and only participants with a score higher than 60 (thus attaining the threshold for the presence of a high level of alexithymia) were analyzed. TAS-20 scores ranged between 61 and $73($ mean $=64.2)$ in the present sample. This sample was extracted from a larger pool of participants who took part in a larger study. Although not a part of this qualitative study, some participants who were included in the larger study had very low alexithymia scores (below 30 on the TAS-20) and were also interviewed in order to obtain a description of a relational event involving the experience of sadness and joy. In order to contextualize the present findings, transcripts derived from interviews with three individuals with low levels of alexithymia will be used for comparison purposes when discussing the results. The three low-alexithymia individuals were all women, aged between 20 and 23 years, and their TAS-20 scores ranged between 24 and 29.

\section{Instruments}

The 20-item Toronto Alexithymia Scale (TAS-20). The TAS-20 is the most commonly used instrument for measuring alexithymia (Taylor et al., 2016). This selfreport questionnaire has demonstrated adequate validity and reliability, as demonstrated by a Cronbach alpha of .81, a test-retest reliability within 3-week 
intervals of $\mathrm{r}=.77$, an adequate pattern of correlations between factors, and an adequate convergent validity (Bagby \& Taylor, 1999b). A French translated version with similar psychometric properties (Loas, Otmani, Verrier, Fremaux \& Marchand, 1996) was provided to participants in the present study. The total score of the TAS-20 is based on three subscales. The first, called the "difficulty identifying feelings" scale, is used to assess the level of affect representations (e.g., "I am often confused about what emotions I am feeling"). The second, referred to as the "difficulty describing feelings" scale, is used to assess the capacity to find words in order to communicate one's emotional experiences to others (e.g., "It is difficult for me to find the right words for my feelings"). The last subscale, termed the "externally-oriented thinking" scale, is used to measure individuals' tendency to focus their attention and consequently, their level of interest outside of their internal emotional life (e.g., "I prefer to analyze problems rather than just describe them" reversed). In this study, the total score was used to assess the overall level of alexithymia. Participants considered to have high levels of alexithymia had a global score equal to or higher than 61 , in accordance with predefined cut-off scores (Bagby \& Taylor, 1999b).

\section{Procedure}

In the context of a larger study, participants first had to complete online questionnaires for about 75 minutes (which included the TAS-20). They were then contacted by email for an interview, for which they received a compensation of $\$ 15$. Interviews began with participants being asked to recall a relational event involving a typical experience of sadness and then a typical experience of joy, narratives which were used in the present study. This was followed by an experimental emotional induction task which involved viewing only a sad excerpt from a movie. The study ended with a post-induction interview. All interviews were recorded and were later transcribed. The interview segment used in this study, from the initial phase of the larger study, started with the following statement: "I would like you to give me an example of a specific relational exchange that made you feel sad, a typical example that illustrates well your usual experience of sadness". The interviewer then used reformulation strategies and reflections to further explore and specify the participants' word choices. A similar statement was used for the joyful event, which was read as follows "I would like you to give me an example of a specific relational exchange that made you feel joyful, a typical example that illustrates well your usual experience of joy".

\section{Analysis}

An interpretative phenomenological analysis (IPA) approach was used to analyze the data. This type of analysis focuses on the individual experience of each participant. It allows a better understanding of how participants make sense of their experiences. Grounded in a constructivist paradigm, this analysis takes into account a process in which the interpretations of both the researcher and the participant are involved. This analysis follows 6 specific steps. The first step consists of reading the transcript of one participant several times to allow the researcher to become thoroughly familiar with the content of the original data. For the second step, notes on the semantic content, the language used, and the voiced concepts are added to the verbatim. During the third step, emergent themes are developed. The fourth step consists of seeking 
overlapping themes within the added notes. The fifth step is a repetition of the first four steps for each of the remaining participants. Finally, the last step involves the examination of patterns emerging within participants' expressed themes (Smith et al., 2009).

\section{Results}

Data from the interviews revealed six themes associated with sadness and three themes associated with joy. The themes reflected how sadness and joy are phenomenologically experienced but they also permitted to draw inferences about the relative levels of symbolization of emotions. All themes were present in the majority of the participants' discourses. The themes associated with sadness were (1) avoiding contact with sadness, (2) pragmatic sharing, (3) lack of engagement in a resolution process, (4) emotions as the result of external events, (5) confusion with anger and (6) perceived difficulty in identifying and expressing emotions. For joy, although a smaller number of themes emerged, they still suggested a distinct form of symbolization linked to this positive emotion, probably associated with alexithymia. These themes were (1) achievement of goals, (2) expression through action and (3) joy experienced as a shared emotion.

\section{Sadness}

1. Avoiding contact with sadness. Participants described sadness as an affect that they wished to avoid rather than as a subjective inner phenomenon specific to certain relational events. When asked to elaborate on their way of experiencing sadness, participants had a tendency to describe strategies used to minimize their experience of sadness, as well as contact with or awareness of it. Among strategies to decrease contact with sadness, participants talked about evacuation and suppression. First and foremost, participants who seemed to use a suppression strategy talked about "burying", "repressing" or "eliminating" sadness. For participants, this attempt to minimize contact with sadness seemed necessary in order to regulate and handle events surrounding the affect, as if the affect was considered too dangerous or painful to be integrated into psychic life.

P4: I repress it, I think about other things. [...] but, if it's not that, I'll either... um, either, um... It's either self-mutilate, or... to let it pass. [...] I repress it, I listen to music. So that's it, I disconnect myself a little to let it pass.

P1: I was telling myself that this girl, I'll see her again in two of my courses plus the sport that I am training in, so I needed to keep in mind that that I still wanted to keep some kind of good relationship with her, so to eliminate as much as possible my negative feelings.

Several suppression strategies were used by participants. Some also expressed a need to concentrate on other tasks or activities. This need to be "focused" or to "think" about other things denotes a tendency to decrease awareness of the emotion. Others simply talked about eliminating the affect rather than ignoring it. 
Another strategy mentioned by participants aimed at reducing contact with sadness is evacuation. The affect was described as something that needed to be "evacuated" or "taken out" of themselves. As for suppression, this means of rejecting affect seemed to be a method for participants to regulate negative affects when they emerged. This strategy seemed to be used by participants who did not know how to experience sadness otherwise.

P9: I don't have a phase where I lie flat in my bed moping for a week. [...] Well, you know, of course, of course I'm sad inside myself but... That's how I evacuate it. Of course, that's sad but um... you know, I don't know I don't care! (laughs). I'm sorry, but for me, that's how I manage.

2. Pragmatic sharing. This second theme was linked to the first since it also demonstrated that participants indirectly avoided engaging in behaviours that may lead them to come in contact with their feelings. Indeed, even if seven participants out of nine chose to share what happened with someone, they exclusively shared events or facts. They did not mention their experienced affects or their inner states, which enabled them to decrease their contact with it. This finding evokes the operative thinking aspect of alexithymia. Indeed, the content that was shared had a concrete rather than a subjective tone, suggesting that an inner mental space to symbolize emotion was lacking.

P3: I expressed [the fact of being sad for leaving everything behind] by explaining why I had to go. Why I had to rebuild my life and all.

P8: I didn't say to my friend that I was feeling sad, I don't say how I feel but I tend to talk about the events.

3. Lack of engagement in a resolution process. The third theme that emerged was also strongly linked to the first, as it emphasized some sort of avoidance of sadness, whether it be a defense mechanism or the result of a feeling of helplessness when confronted with the emotion. Indeed, when participants tried to explain how the relational event that generated sadness ended, they did not engage in a process aiming to resolve the situation. Results show two strategies used by the participants when a resolution of the sad situation was explored: they either let time pass by without acting, or they severed the relationship linked to the affect by detaching completely from others, or through self-isolation.

On one hand, the tendency to rely on time or to ignore what had happened denoted a certain level of passivity in addressing the emotional situation. The participants' discourse showed that emotions were experienced as imposed on them and that they didn't feel that they could act upon it.

P7: I would say that it's just time you know. Just let my frustration pass, and um, because actually nothing could have eased my frustration, it's just really time. To say to myself, too bad, it's done, move on.

P5: How it ended, there's not much to do, so I accepted it and that's it.

P1: I just understood that I needed to move on to something else.

Language and Psychoanalysis, 2018, 7 (1), 62-83.

http://dx.doi.org/10.7565/landp.v7i1.1583 
In these excerpts, participants addressed the necessity (i.e. "need") they had to stop thinking about the event and to leave it behind them, suggesting that affects were avoided and could hardly be accepted and integrated.

On the other hand, some participants tried to avoid their affects by decreasing or breaking all ties to the individuals that were part of the relational event responsible for the sadness. Even if these participants undertook an action, this strategy was also experienced as an imposed necessity. Furthermore, the strategy didn't allow individuals to engage in a resolution process that would support an adequate regulation of the experienced affect.

P7: I isolated myself, I would always take long walks [...]. It seemed that I had to be alone because it was as if others had betrayed me.

P8: I cut-off all contact, I said okay, I have to move forward. [...] well, too bad and I have to forget about this.

Avoiding to engage in a resolution process seemed to denote that sadness was perceived by participants as a state on which they could not act. It also meant avoiding contact with the emotion.

4. Emotions as the result of external events. The fourth theme added to the third, for it also showed that affects were perceived as a state that cannot be acted on, as imposed by events, as if the participants had been spectators watching them unfold. Indeed, participants described a series of events concretely and concluded that these events had saddened them. Affects were thus perceived as having an external cause triggered by oneself or others and these events "inflicted" sadness upon them. Affects and the subjective aspects pertaining to the events were not addressed, suggesting a lack of integration of the emotion into the participants' experience.

P8: What made me feel the most sadness is when I talked to my best friend again [who doesn't live in the same country anymore].

P3: [...] in the end, it's sad to quit, to quit everything to make sacrifices. [...] It's the unknown that makes me sad to quit everything.

P4: But he doesn't accept it, and that is what makes me sad.

5. Confusion with anger. Although a defining feature of alexithymia is a difficulty in labelling feelings, participants in this study identified a wide variety of feelings associated with sadness. Among them, anger was expressed by most participants to various degrees ("discontent", "frustrated", "angry", "enraged").

P4: It's pretty mixed when it happens to me, I'm not too sure between sadness and anger. Both are alternating. It mostly comes after a state of anger that doesn't last long, it gives way to sadness rapidly.

As stated by this participant, the two affects were almost interchangeable for the participants, despite being qualitatively different. Sadness is normally a response to a loss whereas anger is generally considered a response to an event perceived as an 
obstacle or aggression. Nevertheless, participants seemed to experience these two affects concurrently and were unable to adequately distinguish them, exhibiting a lack of differentiation.

6. Perceived difficulty in identifying and expressing their feelings. In addition to perceiving sadness as an affect associated with anger, participants perceived themselves as experiencing only a few emotions, or as having difficulty being in touch with their feelings. They also expressed a difficulty expressing feelings, despite the fact that they labelled several different feelings when describing relational events. This theme was explicitly referred to by participants, and could also be inferred by the difficulty that some participants demonstrated in recalling a typical event associated with sadness.

P6: It's not something that I live a lot, so I don't have a specific example in mind.

P9: I don't know how to describe it, I'm not that close to my emotions.

P7: I experienced... I don't know if it's sadness! I think it is something else.

The lack of contact with their feelings that the participants reported might have led to difficulties with symbolization since by feeling disconnected from their affects, and by subsequently not approaching them directly, participants seemed to have difficulty translating their emotional experiences into words.

\section{Joy}

1. Achievement of goals. For eight of the nine participants, the joy-related event that was recalled was associated with accomplishing a goal, such as obtaining a scholarship or a license, a victory, or the success of an endeavor that seemed impossible. Therefore, the experience of joy was principally associated with successrelated events.

P9: When I received the letter [of acceptance], I was crazy you know, I was crazy!

Joy was perceived as a reaction to an external event without reference to an internal subjective state.

2. Expression through action. When participants were asked to describe an experience of joy, they described a series of actions rather than an internal state.

P6: How I experienced it, well I took her in my arms, I was smiling...

P9: I was jumping to the ceiling, I went to spend, like my God my entire paycheck you know.

P8: We were in a cafe, we were screaming, we were standing, we were singing and clapping our hands. [...] We had a big smile that we could not take off our face. 
The description of actions could have denoted a lack of symbolization when joy was described solely in terms of behaviour without attention to an internal subjective realm.

3. Joy experienced as a shared emotion. Finally, joy was reported by all participants as experienced with others, who were also experiencing a moment of joy. One of the key components of the participants' discourse was sharing the affect with others. Knowing that the affect was shared and also lived by others seemed to accentuate the subjective experience of joy.

P1: First, you know, a night out with friends, just like that, it's a happy thing but... More than that, the event it, it brought something more, that's it, it created a, like that exactly, like an euphoria, uh, everyone, from what I thought, had a lot of fun

P6: You know, everyone was super happy for her really, it was like, uh, really super fun to see everyone happy [...]. I think that her joy was, um, her joy and her expression of her joy was contagious too.

\section{Discussion}

\section{Individual Differences}

The results of this study provide a snapshot of the emotional discourse of highalexithymia individuals associated with sadness and joy. Furthermore, this study allows for a better understanding of the level of symbolization of emotions exhibited by high-alexithymia individuals. In contrast to the initial description made by Sifneos (1994), student participants with high alexithymic scores on the TAS-20 do not show an extreme deficit in the variety of their emotional representations. Even if participants manifested important inter-individual differences, the majority of participants were capable of naming a number of feelings and of explaining a related, emotionally-laden event. Despite the use of vague emotional words (e.g., "I felt bad"), these individuals were capable of differentiating between typical emotions, for example sadness, guilt and anger. However, it has to be noted that they were asked to discuss a specific emotional interpersonal event (saddening or joyful). As shown in previous studies, high alexithymic individuals do report some negative feelings in the form of complaints of anxiety and depression, and may also talk about nervousness, agitation, restlessness, irritability, and tension, without nonetheless being able to elaborate on these feelings (Sifneos, 1967). The participants of this study however, demonstrated a higher than anticipated degree of emotional awareness (Lane et al., 1996) and availability of emotional representations, as demonstrated by some elaboration and a more differentiated vocabulary than expected. For example, in order to describe a sadness-related relational event, participants used words such as depressed, overwhelmed, incomprehension, or surprise as the source of distress, reported feeling betrayed or not supported by others, loneliness, disappointment, guilt, and feeling torn. Moreover, they also referred to behavioural expressions, physical sensations, and overall references to emotional schemas, for instance situational references (e.g., "it wasn't sadness as when someone dies" (P5)). Some important individual differences among significantly alexithymic participants in terms of their experience of positive and negative emotions are accurately illustrated by these two participants:

Language and Psychoanalysis, 2018, 7 (1), 62-83. 
P4 (sadness): How I live it, it gets stuck in the chest, well in the thorax and the throat. So it keeps it all stuck in the throat. [...]

P4 (joy): (pauses) Well, I must admit (laughs) that nothing really happens like, it's, it's, it's pretty much neutral for real during that time. [...] So, what happens is I'll do like others do around me, I'll be a little bit like a monkey, I'll imitate the gestures I see around, and then we'll raise our arms and we'll hug.

Although this participant demonstrated a typical alexithymic difficulty in distinguishing between subjective emotional feelings and the bodily sensations that accompany states of emotional arousal, and in mentalizing emotions, some other alexithymic participants were capable of a much more elaborate symbolization of emotion, even with similarly high, or even higher, alexithymic scores (P4 $=62, \mathrm{P} 6=$ 73). Indeed, in the two contrasted excerpts, the participant that received the highest score on the TAS-20 has a discourse less typical of high levels of alexithymia:

P6 (sadness): [talking about the loss of her family cat] yeah I was sad because, uh, it was, it was still a presence [...] it occurred with another event in my life where I was hospitalized [...] it changed like all the family dynamics [...]. Um, it was a little bit like a separation, it's not, you know, it wasn't dramatic like you know what I mean, it wasn't the end of the world but at the same time, um, well, I was, yeah, I was sad that he was gone yeah. [...]

P6 (joy): It was a happy event, like this girl, she had so many hard times the past 10 years and then it was like if recently everything fell into place for her, like um, this joy that she's experiencing, well it's like it made me realize that you know, me too I've had hard times in the last few years and it says that it's not because you've been through difficult things that you'll stay there you know.

This participant was able to attribute some meaning to her experience, in part by associating the event with other significant elements from her past (Lecours et al., 2009). Therefore, the difference between these two participants on symbolizing capacities properly illustrates a more diverse and complex picture of alexithymia than the one suggested by the TAS-20's set of items (Bagby \& Taylor, 1999b). A possible explanation for this difference might reside in the nature of the test itself, a self-report measure. Indeed, during the interview, participants demonstrated an ability to perceive themselves as presenting a difficulty in being in touch with their own affects. However, what constitutes a difficulty can vary largely among individuals based on subjective standards, such as expectations of what being in tune with one's feelings would normally look, and feel like. Perception of self is a crucial determinant of selfreport measures, and the variety of subjective standards could explain why different levels of symbolization were noted, despite all participants being considered to have high alexithymia scores according to the TAS-20.

\section{Sadness}

Beyond individual differences, the many experiential themes shared by the participants revealed a rather unified high-alexithymia picture when sadness is concerned. Even if alexithymia is conceptualized as probably involving both ability

Language and Psychoanalysis, 2018, 7 (1), 62-83. 
deficits and avoidant defenses (Preece, Becerra, Allen Robinson \& Dandy, 2017), the subjective narratives of sad events, as reported by high-alexithymia individuals seem to indicate a defensive pattern of avoiding negative emotions. Based on the participants' interviews, the access, to some extent, to a feeling schema including behavioural and situational components, seems to indicate at least some cognitive abilities in the treatment of affect. From a more subjective perspective, the narratives indicate that sadness seemed to be experienced but avoided, leading to a truncated elaboration process (see Figure 1).

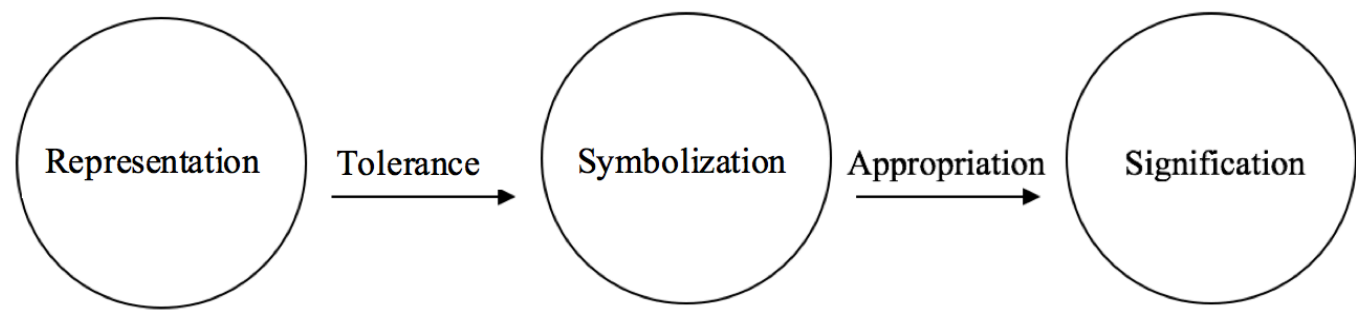

Figure 1

Normal sequence of the emotional elaboration process.

In the normal sequence of this elaboration continuum, the symbolizing capacities become more complex as the level of elaboration increases. The capacity for symbolization allows for increasing containment of the affect, which thus permits a more complex elaboration. However, high-alexithymia individuals report the subjective need to avoid sadness, which may restrict their tolerance of it, which may consequently constrict the elaboration process. Therefore, instead of depicting alexithymia as a deficit that prevents symbolization, the way high-alexithymia individuals experience sadness suggests that the first steps to symbolizing would be accessible but seldom attained as a result of their subjective need to avoid sadness, hence resulting in a defensive avoidant attitude. However, whether a defense or deficit played a role in the limited elaboration process could not be assessed by the present data.

At the lowest level of elaboration, to "represent" refers to the capacity to characterize various angles or components of an emotion. Throughout their interviews, participants demonstrated an ability to label several standard feelings, in addition to an ability to verbalize somatic (e.g., P4: "it gets stuck in the chest, well in the thorax and throat") and verbal representations (e.g., P3: "in the end, it's sad to quit, to quit everything to make sacrifices"). This reflects the participants' capacity for cognitive representation of the emotion schema. However, the confused perception of anger and sadness suggests an incomplete process of representation. Indeed, two qualitatively different emotions were intertwined, highlighting some difficulties in differentiating among representations of emotions. In an optimal process of elaboration, the presence of representations supports an ability to tolerate emotions. Yet for high-alexithymia participants, the defensive pattern associated with sadness indicates that this feeling is mostly avoided. Indeed, participants adopted a variety of defensive attitudes, suggesting a low level of tolerance. 
First among these attitudes is the description of sadness as an emotion that should be suppressed or evacuated. The "evacuation" of sadness evokes conceptual formulations by Bion who proposed that unelaborated emotions (beta elements) have to be handled mentally by excessive projective identification, which amounts to the mental apparatus getting rid of the concretely painful experience (Bion, 1962). This conceptualization has been used to understand alexithymia by clinical theorists (Graham, 1988). Second is the tendency to avoid engaging in processes that include being in touch with an emotion, such as sharing the feeling with peers or initiating conflict resolution. Lastly, some typical defensive behaviours were also present, such as laughing during highly emotionally charged moments:

P4: Yeah, and then when I talk about it afterwards, well, it makes me want to laugh, it's nervous!

P7: [...] it's that, I wish I could have helped her, but the only thing I was feeling was frustration. I don't know if you ever lived with someone who's anorexic, but it's really um... you just to shake her and say eat damn it! (laughs)

Therefore, the defensive attitude adopted by participants hinders tolerance, thus inhibiting the capacity to link representations together in order to symbolize them. Symbolic thinking refers to the emergent capacity to evoke a referent or an analogy, while associating it to representations, in order to verbally describe emotions, such as by likening emotional representations to a related situation (e.g., P6: "it was a little bit like a separation"; P7: "I needed to be alone because it was as if others had betrayed me"). As shown by the perceived difficulty in expressing and perceiving feelings, the symbolizing capacities demonstrated by the participants of this study seem to be weak and limited, due to their avoidance tendencies.

Since symbolization facilitates appropriation, the latter is also constricted by the defensive attitude described in the subjective experiences of high-alexithymia participants. Subjective appropriation is conceptualized on a continuum, with at one end an unconscious or repressed representation of affect (not-me), and at the other end, the affect is experienced as one's subjective and meaningful internal state (Lecours et al., 2009). However, for the participants in this study, sadness was often experienced as caused by external events, for example, as a consequence of another's actions. Affect doesn't seem to be fully appropriated by the participant (such as expressed with "I", considered as "their own"), suggesting that emotions are perceived as imposed rather than as internal states. The results reveal a lack of subjective appropriation, thereby indicating a lack of underlying symbolization, considering that appropriation normally comes with high levels of mentalization (Lecours \& Bouchard, 1997).

Finally, appropriation in turn allows for meaningful connections, namely enriching the meaning of the emotional experience by linking it to previous life events or other elements of autobiographical memory (Lecours et al., 2009). During interviews, the underdevelopment of the elaboration process, and the subjective necessity to avoid sadness reported by the participants, suggest the presence of limited "meaning making" in their speech. 
Descriptions of reactions from low-alexithymia individuals can allow for a better appreciation of the present results. Excerpts from interviews with low-alexithymia participants taken from the larger study are presented below for comparative purposes. The comparison highlights high-alexithymia individuals' low level of appropriation. Low-alexithymia participants perceive the experienced affect as an internal state and the subjective appropriation is much more elaborated:

PControl(C)1: [...] then um, an example of sadness like that, my visits there don't really happen as I would like and, I feel disappointed when I think, uh, of the quality time I spend with my parents and all that. So, it's not as I expected and I would like it to be otherwise. [...] I'm the kind of person who, um, expresses how I feel and, um, tries to find solutions so that it doesn't happen again, but on the spot, I, uh well, I was a little bit like... hum, I don't know like um... I just felt that... just really disheartened and I felt like doing nothing, I felt discouraged so when it happened it was like a huge down.

PC2: I was sad because I was telling myself that maybe it is me who's not capable of tolerating, like he's new to the job and he tries his best and all and I was thinking maybe it's me that is not capable of interacting well with him, so I was a little bit doubting myself.

These participants are engaged in an introspective process that allows them to be in touch with the contained and appropriated affect in order to regulate it. Contrary to their high-alexithymia counterparts, the discourses of low-alexithymia participants shown above do not reveal signs of defensive attitudes nor deficits in cognitive treatment of emotion and, therefore, indicate that these individuals are capable of a more complex elaboration process. Like their counterparts, low-alexithymia participants demonstrate a capacity for representation through their ability to label several distinctive feelings. However, unlike high-alexithymia participants, they do not demonstrate a confusion with regards to their perception of sadness and anger. Moreover, the presence of improved representations reinforce their ability to tolerate affects, as shown by the tendency of participants to engage in the resolution of conflicts and to reflect on their sadness, rather than avoiding it. Lastly, appropriation of affect is also developed as opposed to the high-alexithymia participants. Indeed, feelings are described as fully one's own subjective states (e.g., I was sad) as opposed to being the result of an external cause.

\section{Joy}

As an emotion, joy seemed to be easier to symbolize than sadness for the participants. All participants were able to easily share stories involving typical events associated with joy. Nonetheless, some aspects of their narratives suggest some kind of difficulty symbolizing. Although the elaboration of joy first appears quite usual and not marginally distorted by high-alexithymia participants, some observations denote a few similarities with the verbal treatment of sadness.

While joy is experienced in situations of accomplishment, the participants do not articulate or symbolize their internal state of pride. Since the experience of joy does not appear to be mediated by pride, a difficulty in distinguishing between qualitatively

Language and Psychoanalysis, 2018, 7 (1), 62-83. 
different feelings is suggested, as well as some deficit in representing affects. This characteristic evokes the difficulty in distinguishing between sadness and anger when describing the relational events associated with sadness. Next, situations of success are often the result of external events such as a victory in a sporting event, obtaining a scholarship or a successful test result. This hints at the emotional experience associated with sadness described by the participants, which are also understood as caused by events. As with sadness, such an externalization of causality suggests a limited appropriation of the subjective experience of joy. Finally, participants described their joy experience with behavioural terms instead of internal states, which also points to a low level of internalization or appropriation. This last characteristic, in addition to indicating a lack of subjectivation or mentalizing, is congruent with the pragmatic thinking associated with high levels of alexithymia. However, some research indicates that positive emotions might be naturally more easily expressed through behaviour (Mortillaro, 2018), which would mitigate the interpretation of joy being less mentalized when expressed with gestures.

When the joy narrative of alexithymic participants is compared to their lowalexithymia counterparts, the tendency to associate joy with external events becomes more clearly characteristic of high levels of alexithymia. During interviews, the discourse of low-alexithymia individuals is more pronouncedly directed towards interpersonal intimacy or general well-being, themes associated with less outward or behavioural manifestations of joy (e.g., screaming, dancing, hugging, etc.). Moreover, the low-alexithymia participants had a greater tendency to describe an internal state, rather than concrete actions, than high-alexithymia participants:

PC3: Of course when I like like, I read a lot of novels and you know, I let myself go a lot, let's say I get absorbed by the character's emotion like, but that doesn't count, that, that doesn't work, no, I don't know, just everyday joy let's say I'm happy sometimes just when my mom cooks a good meal, I'm happy to come home and... [...] And well you know I'm happy inside of me like.

PC2: We were about to go to sleep and all and we were talking like in an intimate moment and he was like very, very sweet and very kind and you know I felt really good being with him, I felt we were close and all, and that made me really happy like I felt like, uh, good to be with him and, uh, happy that I had a beautiful relationship like that like privileged. [...] like a feeling of well-being like I... I had the impression that like with him everything would be okay, you know, like a feeling that... like life is beautiful and you know like general and, uh, maybe, uh, uh, hmm, ease.

To conclude, the differences between the elaboration of joy and sadness show that despite some similarities, the symbolization of positive and negative emotions is not equivalent. First, the lack symbolization of positive emotion is not associated with deficits in coping or emotion regulation. High-alexithymia participants do not report the subjective need to avoid feelings of joy, therefore it appears that participants do not adopt a defensive strategy with regards to this emotion. Rather, they tend to share and experience this feeling with others rather than to avoid it as they do with sadness. This also suggests a good level of tolerance of the emotion. However, symbolization, appropriation and signification levels are rarely reached in the elaboration process, as manifested in the high-alexithymia participants' discourse. Despite this, since the low

Language and Psychoanalysis, 2018, 7 (1), 62-83. 
level of elaboration is not associated with emotion regulation difficulties, the manifestations of high levels of alexithymia are somewhat less apparent. On the whole, positive and negative affects are not accessed in the same way by highalexithymia individuals. Nevertheless, our data suggest that the elaboration process is also incomplete for positive emotions. This result could partly be explained by some level of anhedonia, since alexithymia and trauma can be related, as described by Krystal (1981). Some studies have demonstrated that individuals with high levels of alexithymia frequently report histories of deficiencies in the caregivers' response, which result in a reduced proneness to experience pleasurable emotions (Bagby, Taylor, Parker, 1993). The reduced inclination to experience pleasurable emotions might be explained by the lack of symbolization, appropriation and signification of joy. However, it has to be noted that the participants do not subjectively report a difficulty or a reduced proneness to experience joy.

\section{Limitations of the Present Study}

This study has some limitations. Firstly, the data treated in a phenomenological stance allows a rich description of the participants' experience, but without fuller data on typical low-alexithymia verbal productions, it does not enable us to assert that this description is specific to high-alexithymia individuals. Secondly, the present sample was recruited in an undergraduate psychology program, which means that the participants might be representative of a group of more articulate high-alexithymia individuals, more characterized by defense than by deficit in the representation and verbalization of emotions. Moreover, their schemas, knowledge, and vocabulary of emotion might be more elaborate than the average population considering their studies. Also, although the TAS-20 is the most widely employed method for assessing alexithymia, a multi-method approach to the evaluation of alexithymia would have produced a more robust identification of participants presenting higher levels of alexithymia. For instance, the present findings indicate that highly alexithymic individuals vary as to their level of symbolization of emotions. The wide interindividual variations found in the highly alexithymic individuals in the present study might rest on the mono-method assessment of alexithymia used here. Relatedly, the interview format used in the study was not typical of qualitative studies as it was semi-structured and it asked participants to discuss feelings that are well-defined and delineated. This could have had the effect of favoring a more elaborate approach to emotions. Finally, this study offers a description of high levels of alexithymia manifestations without addressing the underlying construct.

\section{Conclusion}

In conclusion, the present study aimed to provide a better understanding of how highalexithymia individuals describe their experiences of sadness and joy, as well as to describe the major themes found in the emotional discourse of high-alexithymia individuals. In light of how participants reported their subjective emotional experiences, sadness was experienced as an imposed state that needed to be avoided rather than as a subjective state. Joy was also experienced as the result of external events, but was not avoided. The study also suggests that the following verbal strategies could be used to reduce contact with negative affects: experiencing an affect as the consequence of an external event, the tendency to not share emotional content 
with peers and to avoid resolving problems leading to emotional events. These strategies, used defensively, could hinder the capacity to contain and elaborate affects and therefore, its symbolization. These results suggest a difficulty with integrating affect into psychic life. Nevertheless, the high-alexithymia functioning that emerges from the present data is much more diversified than what is implied by the clinically based conceptualization of alexithymia. Participants demonstrate a variety of abilities for elaborating sadness. Participants also seem to present some difficulties symbolizing joy, although it appears to be to a lower extent than for the symbolization of sadness. Indeed, high-alexithymia participants discussing joy do not tend to address their internal states and frequently describe affects as the result of external events, suggesting a low level of appropriation. Despite this, positive affects are not avoided and are shared with others, suggesting that there is a difference in the elaboration process between positive and negative emotions. The non-defensive attitude toward joy seems to confirm that symbolization differs according to emotion valence (positive vs. negative) (Lundh et al., 2002, Luminet et al., 2004; Páez et al., 1999; Wagner \& Lee, 2008). This study contributes to the understanding of alexithymia by suggesting that core deficits are found in the actual verbal expression and elaboration of emotions in highly alexithymic individuals, although theses deficits appear to be more important when dealing with negative emotions such as sadness. Our findings also suggest that various levels of alexithymia severity can be found in the emotional discourse of highly alexithymic individuals.

\section{Acknowledgement}

This research was made possible by an APsaA Fund for Psychoanalytic Research grant (2009-2010).

\section{Authors' Biographical Note}

Marie-Mathilde Dupont-Leclerc is a Ph.D. candidate in research and clinical psychology at Université de Montréal. She is a member of the Laboratoire d'Étude sur la Régulation des Affects et la Psychopathologie (LÉRAP). Her research interests include alexithymia, emotional symbolization and psychopathology. She is interested in how individuals make sense of their emotional experiences and how their subjective narratives shape their emotional functioning.

Serge Lecours, Ph.D., is full professor at the Psychology Department of the Université de Montréal. He is also a clinical psychologist. He directs the Laboratoire d'Étude sur la Régulation des Affects et la Psychopathologie (LÉRAP). He does research on emotional dysegulation and how it is associated with psychopathology. His psychoanalytic contributions include a conceptualization of the mentalization of affect that emphasizes its implicit components. His empirical contributions include the creation and application of a measure of mentalization of affect in verbal expression. $\mathrm{He}$ also conducts research on the alexithymia construct. He is particularly interested in the emotional roots of depression and borderline personality disorder. 


\section{References}

Bagby, R. M., Parker, J. D. \& Taylor, G. J. (1994). The twenty-item Toronto Alexithymia Scale-I. Item selection and cross-validation of the factor structure. Journal of Psychosomatic Research, 38, 23-32.

Bagby, R. M., \& Taylor, G. J. (1999a). Affect dysregulation and alexithymia. In J. Taylor, R. M. Bagby, \& J. D. Parker (Eds.), Disorders of affect regulation: Alexithymia in medical and psychiatric illness. Cambridge, UK: Cambridge University Press.

Bagby, R. M. \& Taylor, G. J. (1999b). Measurement and validation of the alexithymia construct. In J. Taylor, R. M. Bagby, \& J. D. Parker (Eds.), Disorders of affect regulation: Alexithymia in medical and psychiatric illness. Cambridge, UK: Cambridge University Press.

Bagby, R. M., Taylor, G. J., Parker, J. D., \& Dickens, S. E. (2005). The development of the Toronto Structured Interview for Alexithymia: item selection, factor structure, reliability and concurrent validity. Psychotherapy and Psychosomatics, 75, 25-39.

Bion, W. R. (1962). Learning from Experience. London, UK: Heinemann.

Bouizegarène, N., \& Lecours, S. (2017). Verbal elaboration of distinct affect categories and Narcissistic personality disorder features. Psychoanalytic Psychology, 34, 278-289.

Graham, R. (1988). The Concept of Alexithymia in the Light of the Work of Bion. British Journal of Psychotherapy, 4, 364-379.

Krystal, H. (1981). Integration and self-healing in posttraumatic states. Journal of Geriatric Psychiatry, 14, 165-189.

Lane, R. D., Lee, S., Reidel, R., Weldon, V., Kaszniak, A., \& Schwartz, G. E. (1996). Impaired verbal and nonverbal emotion recognition in alexithymia. Psychosomatic Medicine, 58, 203-210.

Lane, R. D., Quinlan, D. M., Schwartz, G. E., Walker, P. A., \& Zeitlin, S. B. (1990). The Levels of Emotional Awareness Scale: A cognitive-developmental measure of emotion. Journal of Personality Assessment, 55, 124-134.

Lecours, S. (2007). Supportive interventions and nonsymbolic mental functioning. The International Journal of Psychoanalysis, 88(4), 895-915.

Lecours, S., \& Bouchard, M. A. (1997). Dimensions of mentalisation: Outlining levels of psychic transformation. The International Journal of Psychoanalysis, 78, 855-875.

Lecours, S., \& Bouchard, M. A. (2011). Verbal elaboration of distinct affect categories and BPD symptoms. Psychology and Psychotherapy: Theory, Research and Practice, 84, 26-41.

Lecours, S., Bouchard, M.-A., St-Amand, P., \& Perry, J. C. (2000). Assessing verbal elaboration of affect in psychotherapy: A preliminary report and single case study. Psychotherapy Research, 10, 47-56.

Lecours, S., Robert, G., \& Desruisseaux, F. (2009). Alexithymie et élaboration verbale de l'affect chez des adultes souffrant d'une pathologie respiratoire. European Review of Applied Psychology, 59, 187-195.

Loas, G., Otmani, O., Verrier, A., Fremaux, D., \& Marchand, M.-P. (1996). Factor analysis of the French version of the 20 items Toronto Alexithymia Scale (TAS20). Psychopathology, 29, 139-144. 
Luminet, O., Rimé, B., Bagby, R. M., \& Taylor, G. (2004). A multimodal investigation of emotional responding in alexithymia. Cognition and Emotion, 18, 741-766.

Lundh, L.-G., Johnsson, A., Sundqvist, K., \& Olsson, H. (2002). Alexithymia, memory of emotion, emotional awareness, and perfectionism. Emotion, 2, 361379.

McDougall, J. (1978). Primitive communication and the use of contertransference: Reflections on early psychic trauma and its transference effects. Contemporary Psychoanalysis, 14, 173-209.

Monsen, J. T., Eilertsen, D. E., Melgård, T., \& Ødegård, P. (1996). Affects and affect consciousness: Initial experiences with the assessment of affect integration. Journal of Psychotherapy Practice and Research, 5, 238-249.

Mortillaro, M., \& Dukes, D. (2018). Jumping for Joy: The Importance of the Body and of Dynamics in the Expression and Recognition of Positive Emotions. Frontiers in Psychology, 9.

Páez, D., Velasco, C., \& González, J. L. (1999). Expressive writing and the role of alexythimia as a dispositional deficit in self-disclosure and psychological health. Journal of Personality and Social Psychology, 77, 630-641.

Preece, D., Becerra, R., Allan, A., Robinson, K., \& Dandy, J. (2017). Establishing the theoretical components of alexithymia via factor analysis: Introduction and validation of the attention-appraisal model of alexithymia. Personality and Individual Differences, 119, 341-352.

Roedema, T. M. \& Simons, R. F. (1999). Emotion processing deficit in alexithymia. Psychophysiology, 36, 379-387.

Sifneos, P. E. (1967). Clinical observations on some patients suffering from a variety of psychosomatic diseases. Acta Medicina Psychosomatica, 21, 133-136.

Sifneos, P. E. (1994). Affect deficit and alexithymia. New Trends in Experimental \& Clinical Psychiatry, 10, 193-195.

Smith, J., Flowers, P., \& Larkin, M. (2009). Interpretative Phenomenological Analysis: Theory, Method and Research. London, UK: Sage Publications.

Subic-Wrana, C., Bruder, S., Thomas, W., Lane, R. D., \& Köhle, K. (2005). Emotional awareness deficits in inpatients of a psychosomatic ward: a comparison of two different measures of alexithymia. Psychosomatic Medicine, 67, 483-489.

Taylor, G. J., \& Bagby, R. M. (2013). Alexithymia and the five-factor model of personality. In T. A. Widiger, \& P. T. Costa, Jr. (Eds.), Personality Disorders and the Five-Factor Model of Personality, 193-207. Washington, DC:

American Psychological Association.

Taylor, G. J., Bagby, R. M., Kushner, S. C., Benoit, D., \& Atkinson, L. (2014). Alexithymia and adult attachment representations: Associations with the fivefactor model of personality and perceived relationship adjustment. Comprehensive Psychiatry, 55, 1258-1268.

Taylor, G. J., Bagby, R. M., \& Parker, J. D. (2016). What's in the name 'alexithymia'? A commentary on "Affective agnosia: Expansion of the alexithymia construct and a new opportunity to integrate and extend Freud's legacy". Neuroscience \& Biobehavioral Reviews, 68, 1006-1020.

Wagner, H., \& Lee, V. (2008). Alexithymia and individual differences in emotional expression. Journal of Research in Personality, 42, 83-95. 
Waller, E., \& Scheidt, C. E. (2004). Somatoform disorders as disorders of affect regulation: a study comparing the TAS-20 with non-self-report measures of alexithymia. Journal of Psychosomatic Research, 57, 239-247.

Wotschack, C., \& Klann-Delius, G. (2013). Alexithymia and the conceptualization of emotions: A study of language use and semantic knowledge. Journal of Research in Personality, 17, 514-523.

Zimmermann, G., Salamin, V., \& Reicherts, M. (2008). L'alexithymie aujourd'hui: essai d'articulation avec les conceptions contemporaines des émotions et de la personnalité. Psychologie Française, 53, 115-128. 